\title{
Simulation of Two-Zone Roller Drafting
}

\author{
By Hidehiko Kyuma, Ikuo Tsuchiya and Yōichi Matsumoto, Members, TMSJ \\ Faculty of Textile Science and Technology, Shinshu University, Ueda, Nagano-ken \\ Based on Journal of the Textile Machinery Society of Japan, Transactions, Vol. 34, No. 8, T154-162 (1981-8)
}

\begin{abstract}
The purpose of the present study is to simulate the effects of doubling and drafting upon sliver irregularity in two-zone drafting of the three-over-three roller system. Two types of slivers, i.e. random and optional slivers were considered. It is assumed that a random sliver is made from fibers of uniform staple length and fineness, and that the number of fiber ends in any short increment of the sliver obeys Poisson distribution. In contrast, it is assumed that the fiber ends in an optional sliver are distributed on the basis of the uniform random numbers ranging in value between 0 and 1 . The effects of the number of doublings, total draft ratio, distribution of draft between the two drafting zones, and the maximum moving distance of the speed change point were calculated on a Y.H.P.9825A personal computer. The results were as follows:

(1) When the maximum moving distance of the speed change point is 0 , the coefficient of variation of a sliver before and after drafting remains unchanged regardless of the number of doublings, total draft ratio and draft distribution. It was found, however, that the drafting changes the power spectrum (Fourier analysis) of these slivers.

(2) When the maximum moving distance of the speed change point is not 0 , the coefficient of variation of a sliver varies with the increase in this distance. The number of doublings, total draft ratio and draft distribution between the two drafting zones considerably influences the power spectrum (Fourier analysis) and the coefficient of variation of produced sliver. It was also found that there is an optimum draft distribution between the two drafting zones.
\end{abstract}

\section{Introduction}

Corley et al. ${ }^{[1-3]}$ studied the optimum draft distribution among drafting zones by conducting experiments using some new type roving frames. Uchida et al. ${ }^{[4]}$ developed a theoretical method of determining the optimum draft distribution in drawing process and Kawabata ${ }^{[5]}$ who developed the theory of random sliver, proved that minimum variation of sliver thickness always exists in drawing process.

In this paper we attemped to simulate the effects of doubling and drafting in the two-zone drafting of three-over-three roller system. One expects that the variation of thickness of sliver produced on a two-zone roller drafting is smaller than that on one-zone roller drafting, if the total draft ratio remains unchanged.

In actual slivers, wave-length of thickness variation is considerably larger than the fiber length, and some fibers are hooked. When this sliver is subjected to doubling and drafting, it can be said that the fiber arrangement in the sliver will be improved, and the variation of thickness will decrease. On the other hand, random sliver was conceived as an ideal model of actual slivers. A random sliver is assumed to be made from fibers of uniform staple length and fineness, and the fiber ends are distributed in an extremely short increment obeying Poisson distribution. It is well-known that the coefficient of variation of random sliver is $1 / \sqrt{N}$ where $N$ is the average number of fibers in cross-section. Therefore, doubling a number of slivers results in a decrease in the coefficient of variation. When the doubled sliver is ideally drafted to the thickness of original component sliver, then the coefficient of variation of the drafted sliver returns to the original value of $1 / \sqrt{N}$ to result in no improvement in evenness. However, the doubling-drafting operation can change the wave-length distribution. In view of above the object of this paper is to numerically simulate how number of doublings, total draft ratio, draft distribution among drafting zones, and the maximum moving distance of the speed change point affect thickness variation.

\section{Program and Assumption}

The simulation was carried out on a Y.H.P. 9825A personal computer. And the simulation program essentially consists of the following four parts:

(A) Program for generating slivers

(B) Program for doubling the generated slivers

(C) Program for drafting the doubled sliver

(D) Program for Fourier analysis of the drafted sliver 


\subsection{Program (A) for Generating Slivers}

The program generates two types of slivers, i.e. random and optional slivers. Let it be assumed that a random sliver is made of fibers of perfectly uniform staple length and fineness, that the fiber axis is parallel to the sliver axis, and that there are no hooked fibers. Futhermore, it is assumed that the variation of thickness of optional sliver is larger than that of random sliver. Other assumptions for generating these slivers are as follows:

1) The number $X[I]$ of fiber ends

The average number of fiber ends $C$ in very short increment $W(\mathrm{~mm})$ in length is given by the following equations:

$$
\begin{aligned}
& C=W D / M L \\
& D=5,315 / N e
\end{aligned}
$$

where $\mathrm{Ne}=$ British count of sliver (s)

$$
\begin{aligned}
& M=\text { fineness of fiber }(\mathrm{d}) \\
& L=\text { staple fiber length }(\mathrm{mm}) \\
& D=\text { denier count of sliver }(d)
\end{aligned}
$$

As shown in Figure 1(a) a sliver is divided into many very short increments of equal length $W$, and every increment includes some number $X[I]$ of fiber ends, $I=1,2,3, \ldots .$. The number of fiber ends in an increment was calculated in the following manner:

(i) Optional sliver

Uniform random number $R[I]$ ranging in value from 0 to 1 and 0.5 in average value is used to generate the number $X[I]$ of fiber ends:

$$
X[I]=2 C R[I], I=1,2,3, \ldots .
$$

(ii) Random sliver

It is well known that the number of fiber ends in random sliver obeys Poission distribution. When the average number $C$ of fiber ends is given by the above equation, the Poisson distribution is determined. Therefore, the number of fiber ends can be generated using the cumulative Poisson distribution curve shown in Figure 2.

(a)

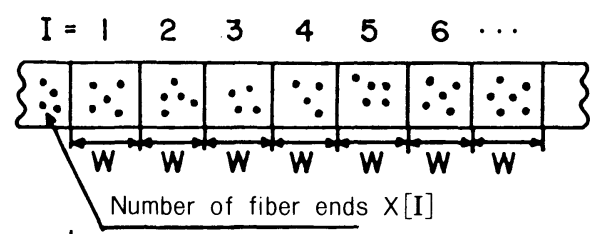

(b)

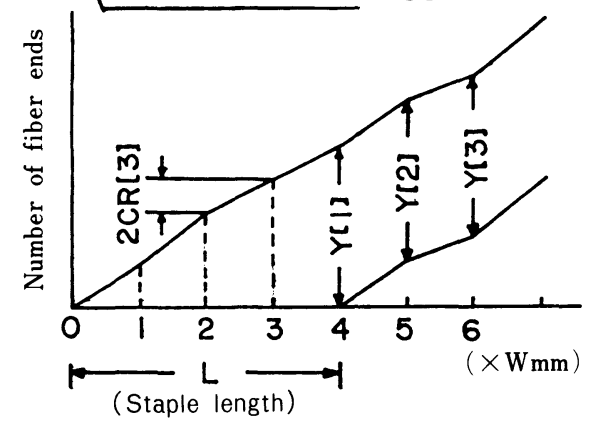

Fig. 1 Number of $X[I]$ of fiber ends and thickness $Y[I]$ of sliver

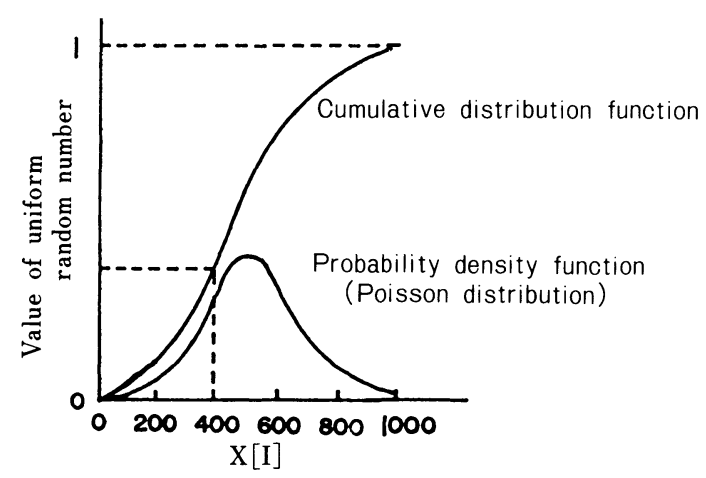

Fig. $2 X[I]$ in random sliver

\section{2) Thickness $Y[I]$ of sliver}

The model shown in Figure 1(b) developed by Watanabe ${ }^{[6]}$ was used to obtain sliver thickness $Y[I]$. The first curve starting at the origin is the cumulative number of fiber ends. The second curve is identical in shape to the first but is displaced rightward by one staple length $L$. Then the sliver thickness $Y[I]$ is given as the vertical distance between the two curves. 3) Sliver length

In view of computer memory restrictions increment length $W$ of $1 \mathrm{~mm}$ and the $I$ range of $1-600$ was used. The whole length of sliver before drafting is, therefore, $600 \mathrm{~mm}$.

Optional or random slivers thus generated are ready for the subsequent doubling action.

\subsection{Program (B) for Doubling the Generated Slivers}

Program (B) doubles the optional or random slivers generated by the above program (A). When the number of doubling is $P$, the number of fiber ends $X_{p}[I]$ in the doubled sliver is equal to the sum of fiber ends in the $P$ slivers.

\subsection{Program (C) for Drafting the Doubled Sliver}

The following assumptions were made in developing pro$\operatorname{gram}(\mathrm{C})$.

1) Random location of speed change point

It was assumed that the random location of speed change point is the cause of draft irregurality. The speed change point was made to be randomly located within the region bounded by a standard position $s$ in Figure 3 suitably located upstream of front roll pair and another point upstrem of $s$ by a distance of $y$. The location $m$ of speed change point is, therefore,

$$
m=R[I] y
$$

where $R[I]$ is the random number discussed above.

2) The movement of fiber ends in one increment

In the first place, it is assumed that all fiber ends in a short increment $W$ are accelerated simultaneously to the front roll speed when the increment reaches the speed change point. As a result, the increment length increases to $Z W$ where $Z$ is the 


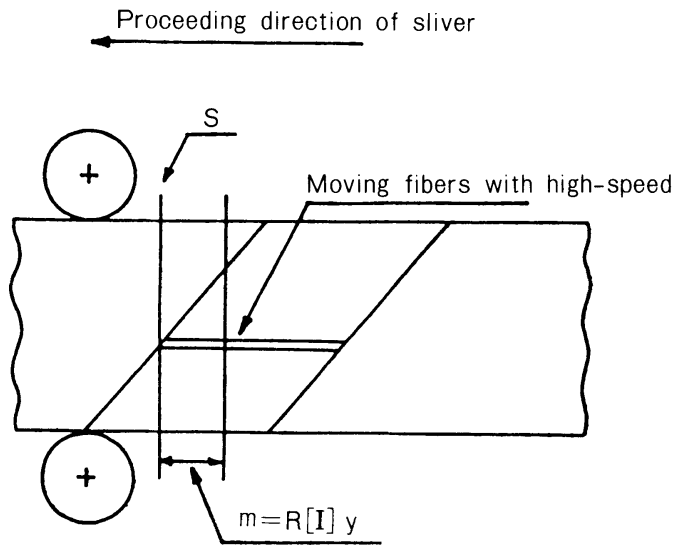

Fig. 3 The movement of the speed change point Standard position of speed change point $=s$ Moving distance of speed change point $=m$

draft ratio. As shown in Figure 4, when the speed change point is located at the standard position, the number of fiber ends in increment $W$ in length remain unchanged by the drafting. However, when the speed change point move away from the standard position, the number of fiber ends in increment $W$ in length changes according to the distance $m$ of the speed change point from the standard position. It is assumed here that this movement of speed change point causes the increase in the variation of thickness of a sliver in drafting. While fibers which were accelerated at the standard position move by a distance of $m$, fibers which were accelerated at position $m$ move by a distance of $m Z$. This results in a relative movement of $m(Z-1)$ between the two groups of fibers. When this quantity is divided by the increment length $Z W$ after the drafting the relative movement $\mathrm{E}$ in terms of the number of increments is obtained:

$$
E=R[I] y(Z-1) / Z W
$$

The number $E$ of increments is in general not an integer to make $E$ expressible in the form:

$$
E=\text { int. }(E)+\text { frc. }(E)
$$

where int. $(E)=$ an integer part of $E$

$$
\text { frc. }(E)=\text { a fraction part of } E
$$

In calculating the number of fiber ends in each increment after drafting, two cases must be considered.

(i) int. $(E) \geqq 1$

In this case, fiber ends $X[I]$ in the $I$ th increment must be added to fiber ends $X[J]$ and $X[J+1]$ in the $J$ th $(J=I-1-$ int. (E)) and $(J+1)$ th increment. Then, the numbers $X[J]$ and $X[J+1]$ of fiber ends after drafting are given respectively by the following two expressions:

frc. $(E) X[I]+X[J]$

$(1-$ frc. $(E)) X[I]+X[J+1]$

Due to this movement, all fiber ends leave the $I$ th increment to make $X[I]=0$. However, when fiber ends in other incre- (a)

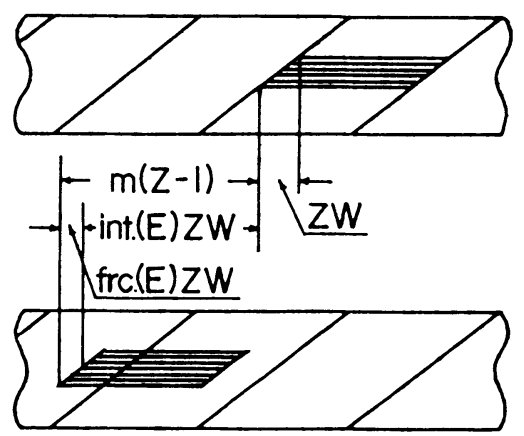

Fig. 4 The sliver after drafting

(a) Position of fibers accelerated at the standard position

(b) Position of fibers accelerated at a position downstream of standard position by distance $\mathrm{m}$

ments make similar relative movements fiber ends are replenished to the Ith increment.

(ii) int. $(E)=0$

In this case, not all fiber ends leave the $I$ th increment. After the drafting the numbers of fiber ends respectively in the $(I-1)$ th and $I$ th increments become:

$$
\begin{aligned}
& \text { frc. }(E) X[I]+X[I-1] \\
& (1-\text { frc. }(E)) X[I]
\end{aligned}
$$

The number of fiber ends in each increment after drafting thus computed enables the sliver thickness at the increment. As the purpose of this paper is to simulate a two-zone drafting, generated slivers pass through this program (C) twice. The increment length increases from $W$ to $Z W$ by the first drafting, making the $Z W$ possibly too long to be regarded an infinitesimal increment length in the second drafting operation. In view of this another program was developed to divide this length $Z W$ into several increments int. ( $Z$ ) in length. As discussed in section 3.5, however, this subdivision of $Z W$ did not bring significant changes. Hence, increment length was made equal to $Z W$ in the second drafting.

\subsection{Program (D) for Fourier Analysis of the Drafted Sliver}

When $x(t)$ is a periodic function of period $T$, power $\rho$ is defined as:

$$
\rho=\frac{1}{T} \int_{0}^{T} x^{2}(t) d t
$$

Fourier series expansion of $x(t)$ is.

$$
x(t)=\frac{a_{0}}{2}+\sum_{n=1}^{\infty}\left(a_{n} \cos \frac{2 n \pi}{T} t+b_{n} \sin \frac{2 n \pi}{T} t\right)
$$

Then the power is.

$$
\rho=\frac{a_{0}^{2}}{4}+\frac{1}{2} \sum_{n=1}^{\infty}\left(a_{n}^{2}+b_{n}^{2}\right)
$$

Where $1 / 2\left(a_{n}^{2}+b_{n}^{2}\right)$ is the power spectrum of the $n$th harmonics.

Program (D) performs the above computations for sliver thickness variation $x(t)$. 


\section{Simulation Results and Discussion}

Simulations were carried out under the following conditions:

Individual fibers have uniform thickness of 1.7 (d).

Individual fibers have uniform length of $30(\mathrm{~mm})$.

British count of sliver is 0.1 (s).

The number of doublings is 4,6 and 8 .

The total draft ratio is 4,6 and 8 .

The maximum moving distance of speed change point is $0,1,2$ and $3(\mathrm{~mm})$.

\subsection{Produced Slivers}

A. Optional slivers

Eight kinds of slivers generated are shown in Table 1. Shown in Table 2 are three kinds of doubled slivers obtained by doubling the slivers in Table 1.

B. Random slivers

Table 3 shows eight kinds of random slivers generated, and Table 4 shows three kinds of doubled slivers made from the slivers in Table 3.

\subsection{Thickness Variation after Drafting}

Figures 5 and 6 show the time variation in the thickness of slivers obtained by drafting the slivers listed in Table 2, III

Table 1 Optional slivers

\begin{tabular}{cccc}
\hline No. & C.V. ${ }^{*}\left(\times 10^{-1}\right)$ & No. & C.V. $\left(\times 10^{-1}\right)$ \\
\hline 1 & 1.04 & 5 & 0.93 \\
2 & 1.02 & 6 & 0.96 \\
3 & 0.96 & 7 & 1.03 \\
4 & 1.08 & 8 & 1.05 \\
\hline
\end{tabular}

${ }^{*} \mathrm{C} . \mathrm{V}$. is the coefficient of variation of a sliver.

Table 2 Doubled optional slivers

\begin{tabular}{rclc}
\hline No. & ND* & Content & C.V. $\left(\times 10^{-2}\right)$ \\
\hline I & 4 & $1+2+3+4$ & 5.56 \\
II & 6 & I $+5+6$ & 3.78 \\
III & 8 & II $+7+8$ & 3.46 \\
\hline
\end{tabular}

*ND is the number of doublings.

Table 3 Random slivers

\begin{tabular}{cccc}
\hline No. & C.V. $\left(\times 10^{-3}\right)$ & No. & C.V. $\left(\times 10^{-3}\right)$ \\
\hline 1 & 5.62 & 5 & 5.00 \\
2 & 6.48 & 6 & 5.65 \\
3 & 4.72 & 7 & 5.53 \\
4 & 5.83 & 8 & 6.25 \\
\hline
\end{tabular}

Table 4 Doubled random slivers

\begin{tabular}{rllc}
\hline No. & ND & Content & C.V. $\left(\times 10^{-3}\right)$ \\
\hline I & 4 & $1+2+3+4$ & 2.93 \\
II & 6 & I $+5+6$ & 1.95 \\
III & 8 & II $+7+8$ & 1.65 \\
\hline
\end{tabular}

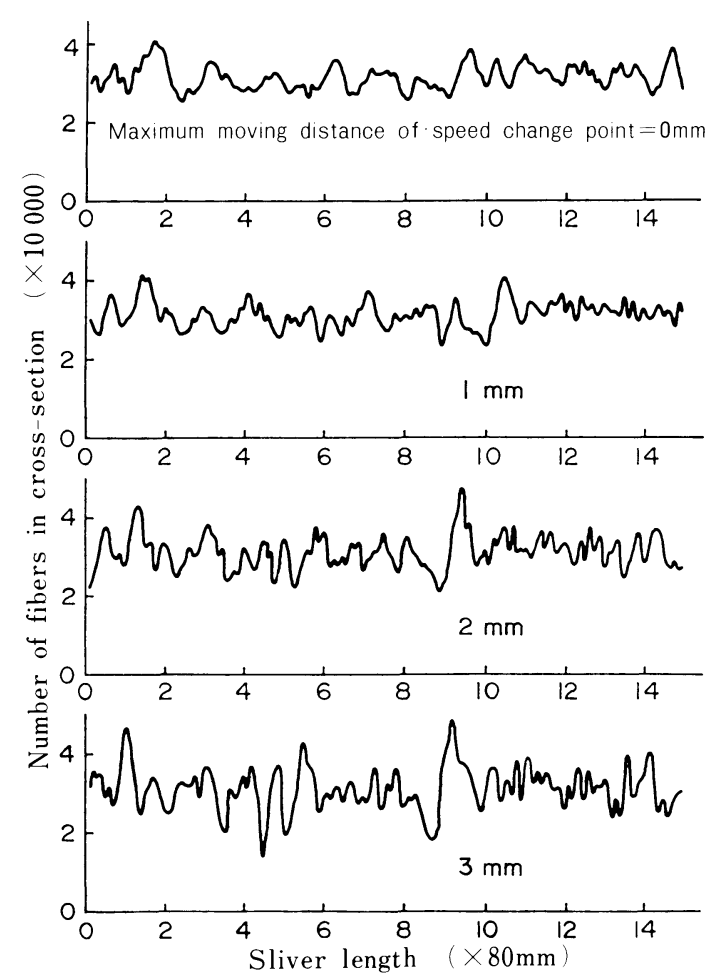

Fig. 5 Thickness variations in optional sliver after drafting under different maximum moving distance $(y)$ of speed change point (Number of doublings $=8$, Draft distribution $=2.5 \times 8 / 2.5$ )

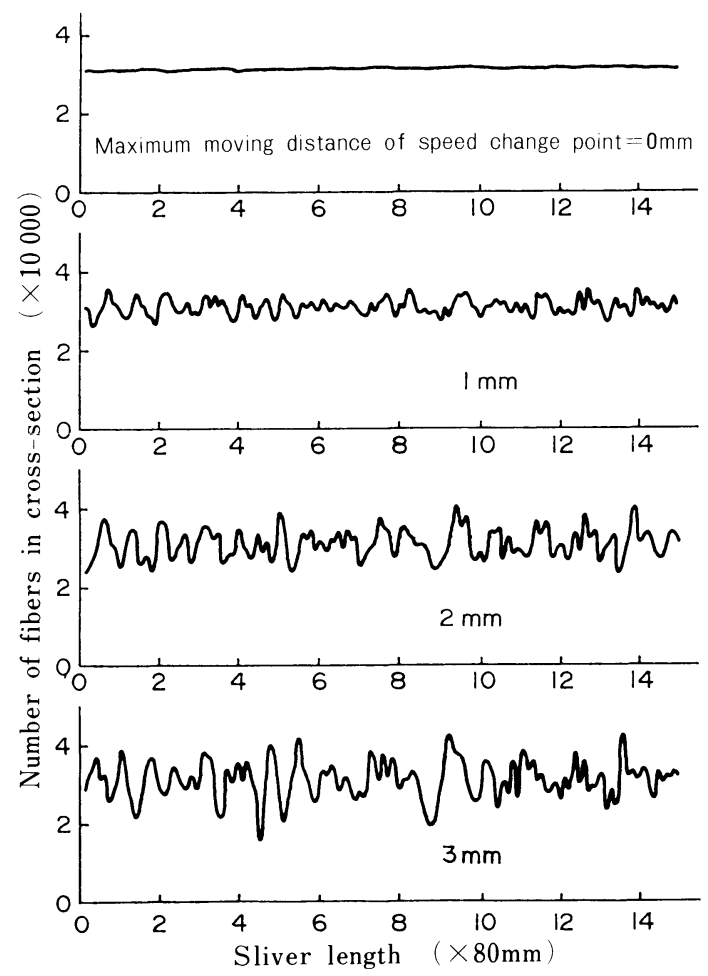

Fig. 6 Thickness variations in random sliver after drafting under different maximum moving distance $(y)$ of speed change point (Number of doublings $=8$, Draft distribution $=2.5 \times 8 / 2.5$ ) 
and Table 4, III respectively. From these figures it can be seen that the variation of sliver thickness after drafting increases with increasing maximum moving distance of speed change point.
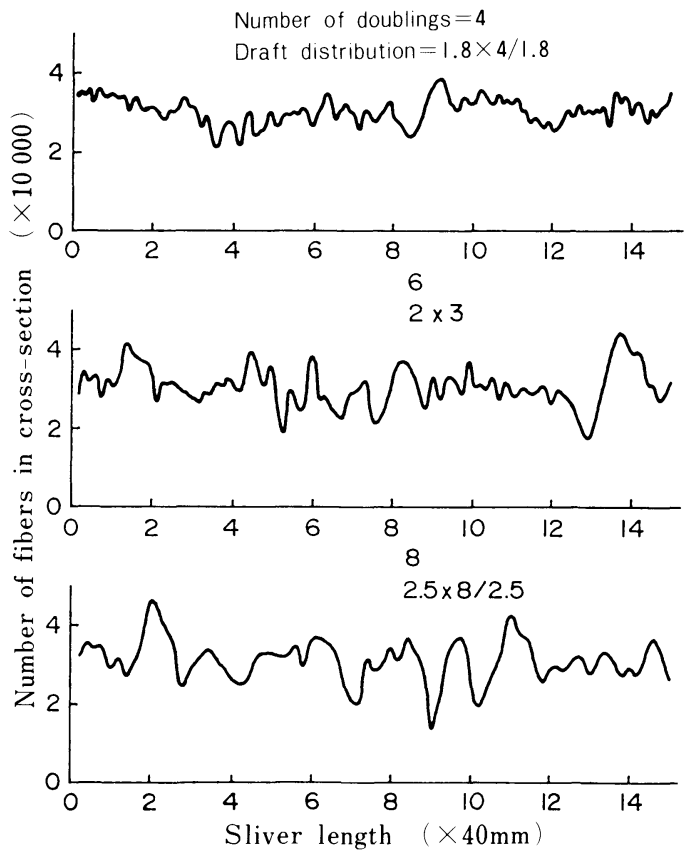

Fig. 7 Thickness variations in optional slivers after drafting (Maximum moving distance of speed change point $=3 \mathrm{~mm}$ )

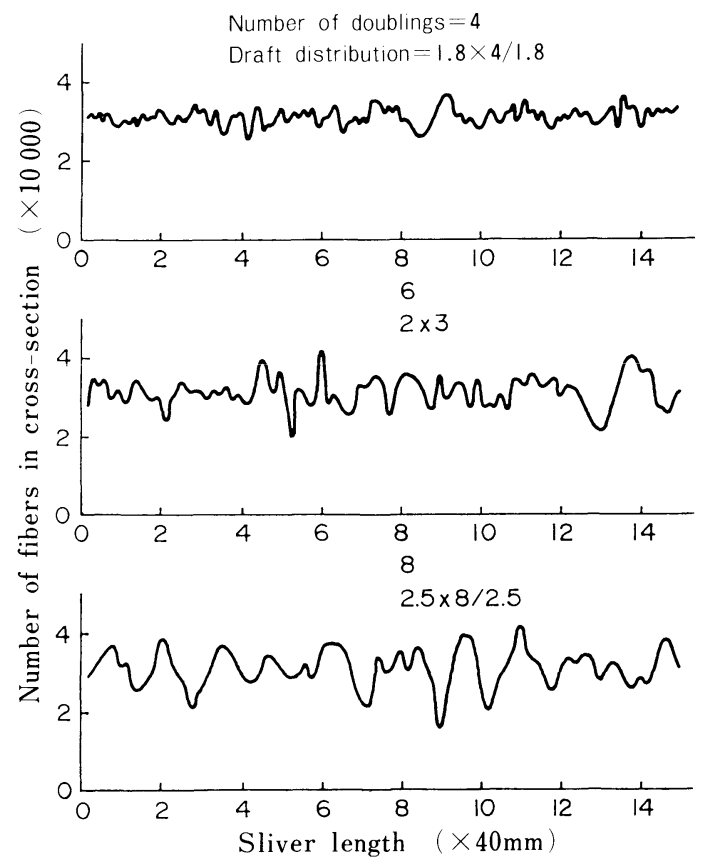

Fig. 8 Thickness variation in random sliver after drafting (Maximum moving distance of speed change point $=3 \mathrm{~mm}$ )
Shown in Figures 7 and 8 are thickness variations in drafted slivers produced from the doubled optional and random slivers listed respectively is Tables 2 and 4. Maximum movement $m$ of speed change point was fixed at $3 \mathrm{~mm}$ and the distribution of draft between the two draft zones were approximately equal. Evidently the thickness variation increases with increasing number of doublings and the resultant increase in draft ratio.

\subsection{Power Spectrum}

Figure 9 shows the square root of the power $\rho$ versus wavelength for the optional sliver in Table 1, I and the random sliver in Table 3, I before doubling and drafting. Both slivers exhibit two prominent peaks in $\sqrt{\rho}$ at wave-length equal to about two and three times the staple length $(30 \mathrm{~mm})$, though the absolute values of the peaks greatly differ by the sliver.

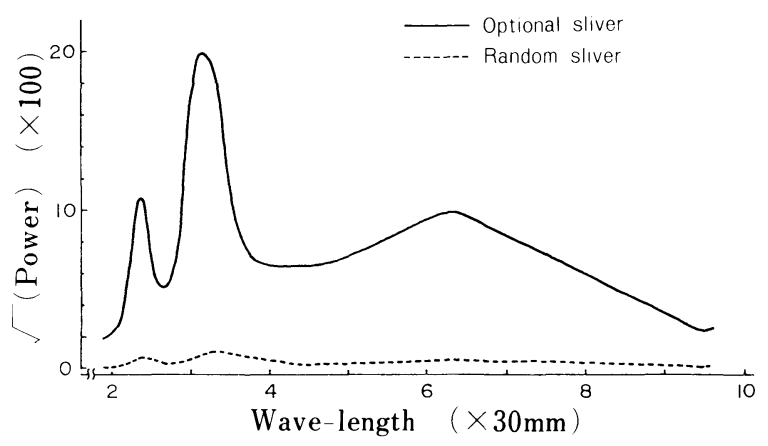

Fig. 9 Square root power spectrum before doubling

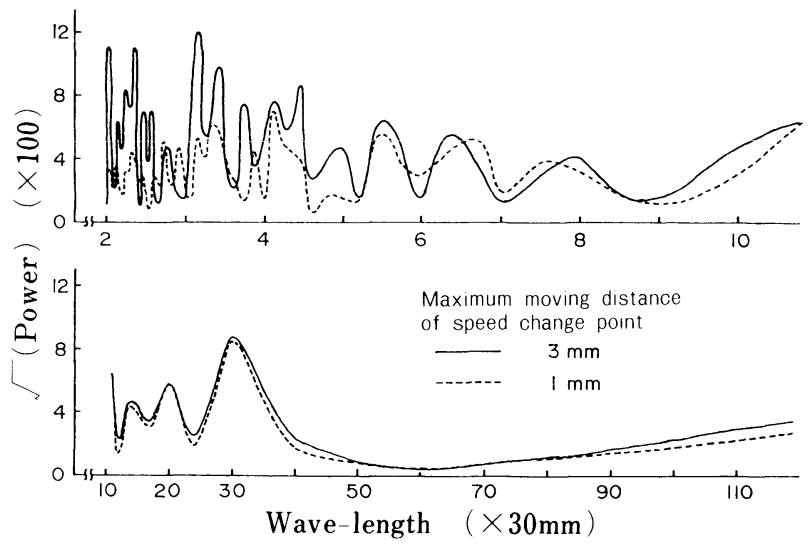

Fig. 10 Square root power spectrum of optional sliver after drafting (Number of doublings $=8$, Draft distribution $=2.5 \times 8 / 2.5$ ) 


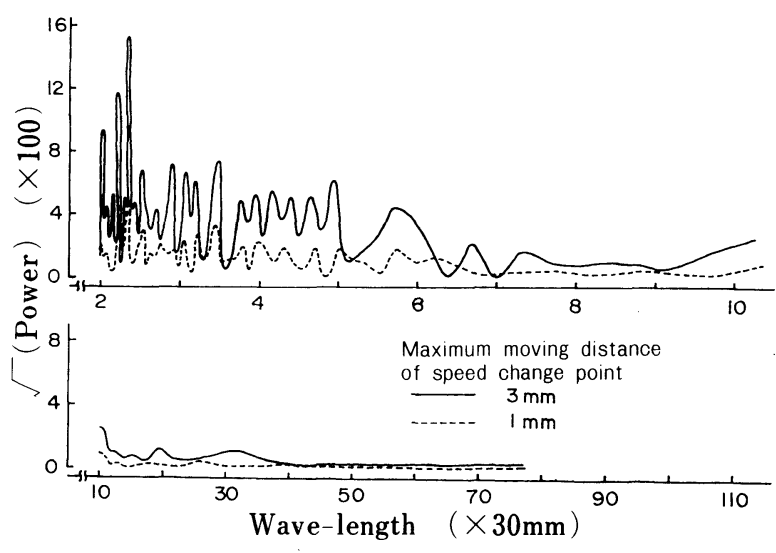

Fig. 11 Square root power spectrum of random sliver after drafting (Number of doublings $=8$, Draft distribution $=2.5 \times 8 / 2.5$ )

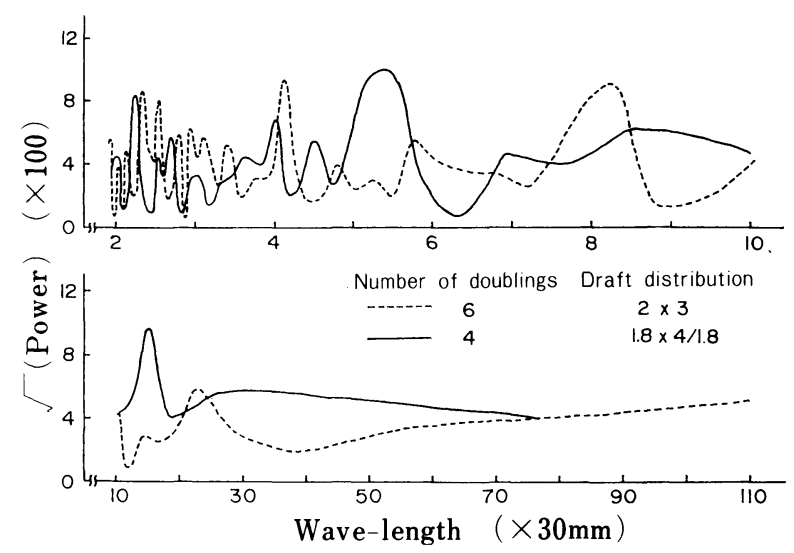

Fig. 12 Square root power spectrum of optional sliver after drafting (Maximum moving distance of speed change point $=3 \mathrm{~mm}$ )

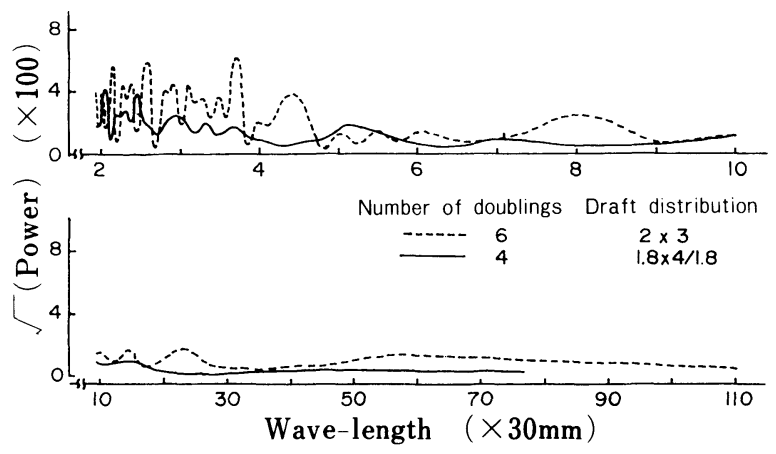

Fig. 13 Square root power of random sliver after drafting (Maximum moving distance of speed change point $=3 \mathrm{~mm}$ )

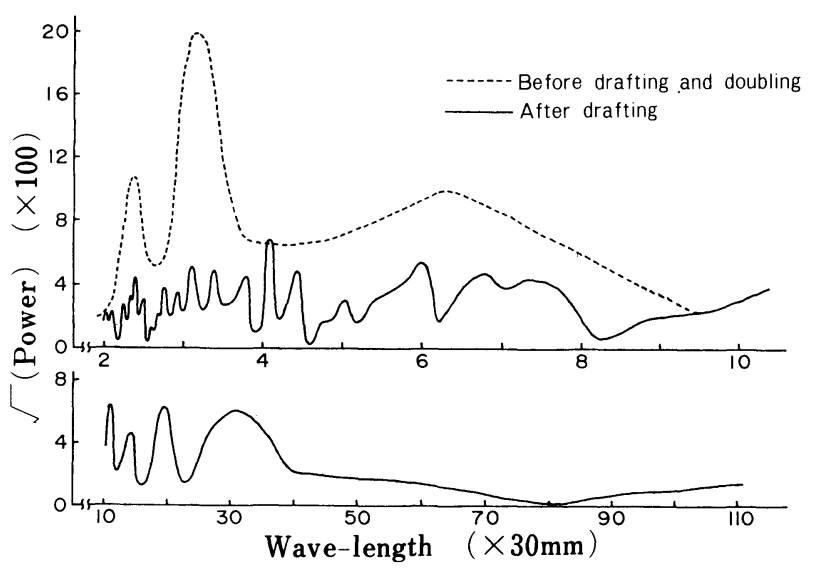

Fig. 14 Square root power spectrum of optional sliver before and after ideal drafting

(Number of doublings $=8$, Drafting distribution $=2.5 \times 8 / 2.5$, Maximum moving distance of speed change point $=0 \mathrm{~mm}$ )
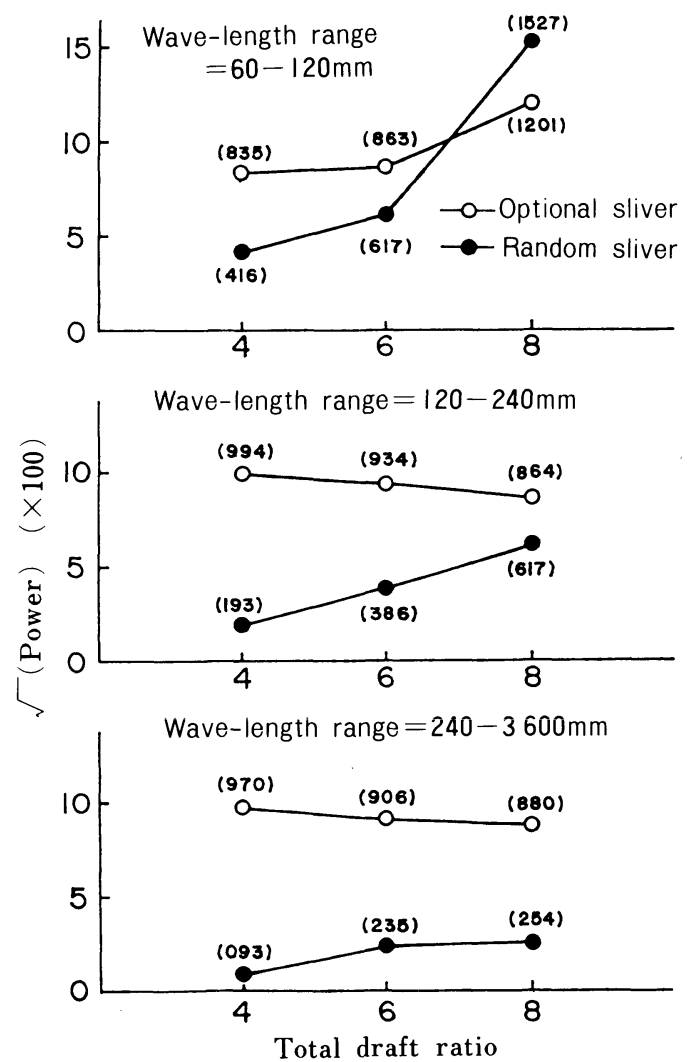

Fig. 15 Maximum value of square root power versus wave-length (The numbers in brackets are maximum values. Number of doublings $=8$, Draft distribution $=2.5 \times 8 / 2.5$, Number of doublings $=6$, Draft distribution $=2 \times 3$, Number of doublings $=4$, Draft distribution $=1.8 \times 4 / 1.8$ ) 
The square root power spectrum shown in Figures 10 and 11 correspond to the slives shown in Figures 5 and 6, and Figures 12 and 13 to the slivers shown in Figures 7 and 8. It may be seen from these figures that the power spectrum is influenced by the number of doublings, the total draft ratio and the moving distance of the speed change point, and that especially within a short wave-length range up to about five times the staple length, there is an increase in the number and intensity of peaks.

Figure 14 compares the power spectrum shown in Figure 9 for an optional sliver before doubling and drafting with that of an ideally drafted sliver made from the doubled sliver listed under Table 2.III. In ideal drafting the maximum moving distance of the speed change point is 0 . As far as the coefficient of variation is concerned the two slivers are both 0.10 to show no effects of drafting but there is a great difference in the wave-length distribution of power.

Figure 15 shows the maximum value of square root power within three different wave-length ranges. In the wave-length range of $60-120 \mathrm{~mm}$ (two or four times the staple length), the maximum $\sqrt{\rho}$ values of both optional and random slivers increase with increasing number of doublings. In both wavelengh ranges of $120-240 \mathrm{~mm}$ and $240-3600 \mathrm{~mm}$, the maximum $\sqrt{\rho}$ values of random stivers increase with increasing number of doublings but that of optional slivers decrease.

In summary it was found that doubling and drafting diminish the prominent peaks in $\sqrt{\rho}$ observed before doubling but the drafting generates new peaks in the short wave-length range of about two or four times the staple length with the new peaks grow with increasing number of doublings and total draft ratio; furthermore peaks extended to increasingly long wave-length range as the total draft ratio increases.

\subsection{The Draft Distribution}

In Figures 16 to 18 the coefficient of variation of slivers obtained by drafting the doubled slivers in Tables 2 and 4 are plotted against draft distribution among drafting zones. The coefficient of variation in every drafted sliver increases with increasing maximum moving distance of speed change point, and has a minimum value at a certain draft distribution except when the maximum moving distance of the speed change point is 0 .

The minimum value of the coefficient of variation shown in Figures 16 to 18 are plotted against the number of doublings (i.e. the total draft ratio) in Figure 19. The minimum value of optional and random slivers increases as the number of doublings and hence the total draft ratio increases to make the drafted sliver more uneven.

Evidently there is an ideal draft distribution that minimizes the coefficient of variation in slivers produced on a two-zone roller drafting.

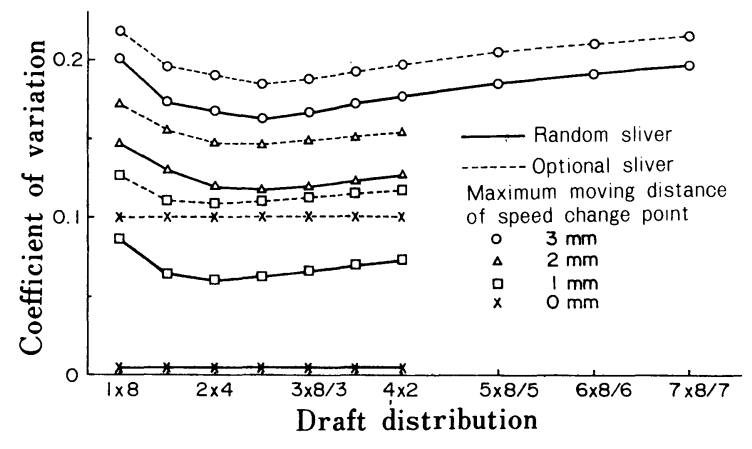

Fig. 16 Coefficient of various versus draft distribution for different slivers

(Number of doublings $=8$, Total draft ratio $=8$ )

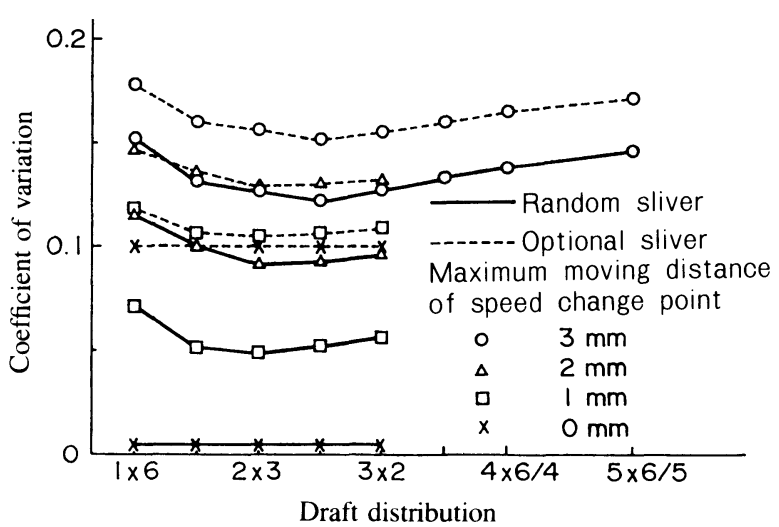

Fig. 17 Coefficient of variation versus draft distribution for different slivers

(Number of doublings $=6$, Total draft ratio $=6$ )

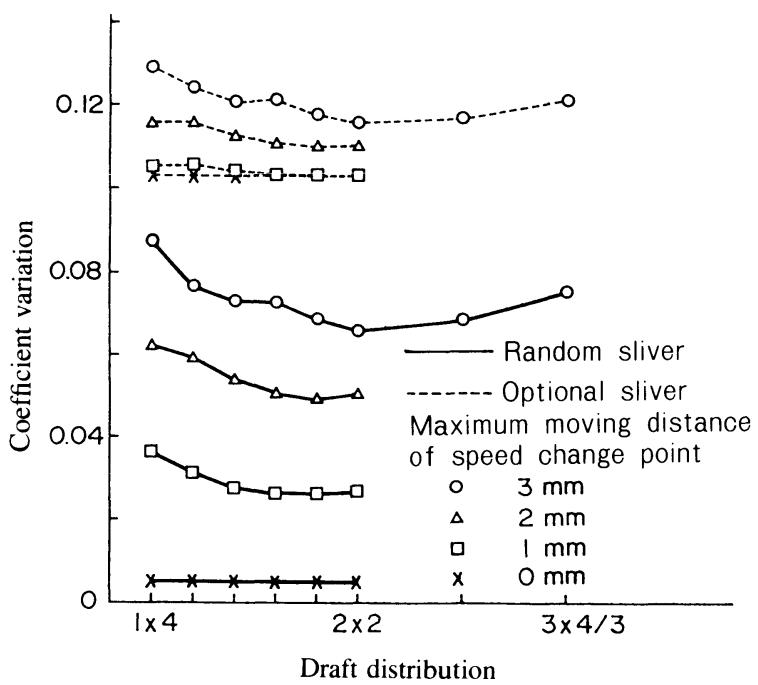

Fig. 18 Coefficient of variation versus draft distribution for different slivers (Number of doublings $=4$, Total draft ratio $=4$ ) 


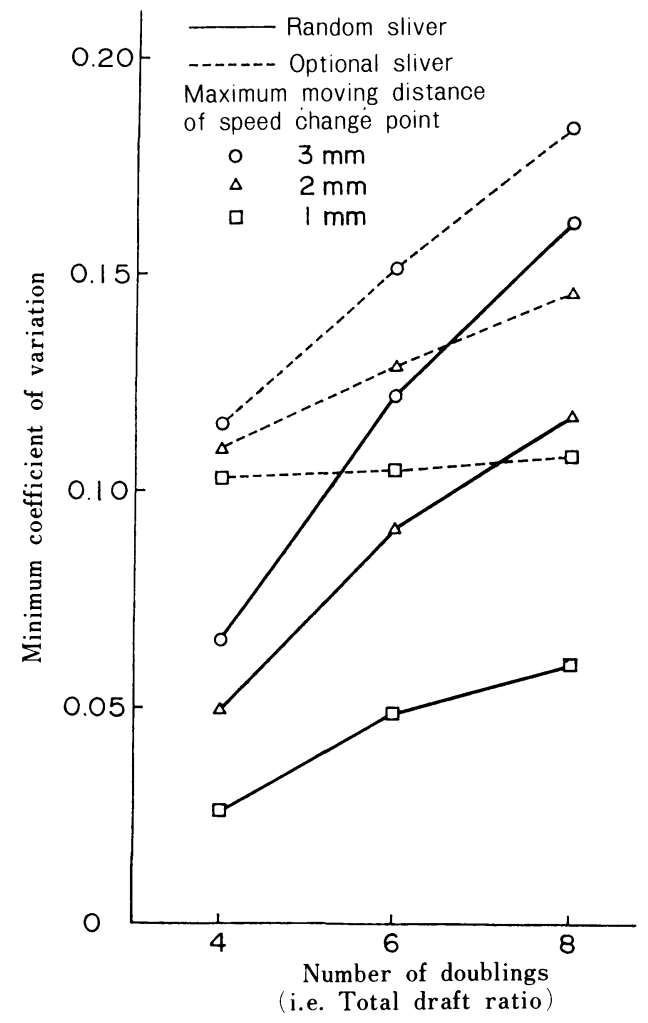

Fig. 19 Minimum value of coefficient of variation versus number of doublings (i.e. total draft ratio) for different slivers

\subsection{Subdividing Length Increments}

As has already been explained in section 2.3 , it is the object of this paper to simulate a two-zone roller drafting; therefore, slivers were drafted twice. The first drafting increases the sliver length increment from $W$ to $Z W$ to possibly make $Z W$ not any more an extremely short increment. For this reason a subdivision of $Z W$ before the second drafting into several increments int. $(Z)$ in length with the fiber ends equally divided was attemped.

Figures 20 and 21 show the thickness variation of optional sliver obtained by drafting the doubled sliver in Table 2, III. The curves shown in Figure 20 are identical to the ones in Figure 5; namely subdivision of $Z W$ was not made in these figures. Figure 21 shows the result of subdividing $Z W$. Except that the curves in Figure 21 contain slight short term variations no great differences are obseved between the two figures.

The square root of the power spectrum in Figures 22 and 23 correspond to the thickness variations in Figures 20 and 21. Here too the subdivision of $Z W$ has little effect.

The coefficient of variation of the sliver in Table 2, III after drafting with and without the subdivision are plotted against draft distribution in Figure 24. The subdivision of $Z W$ does not change the curves greatly nor does if change

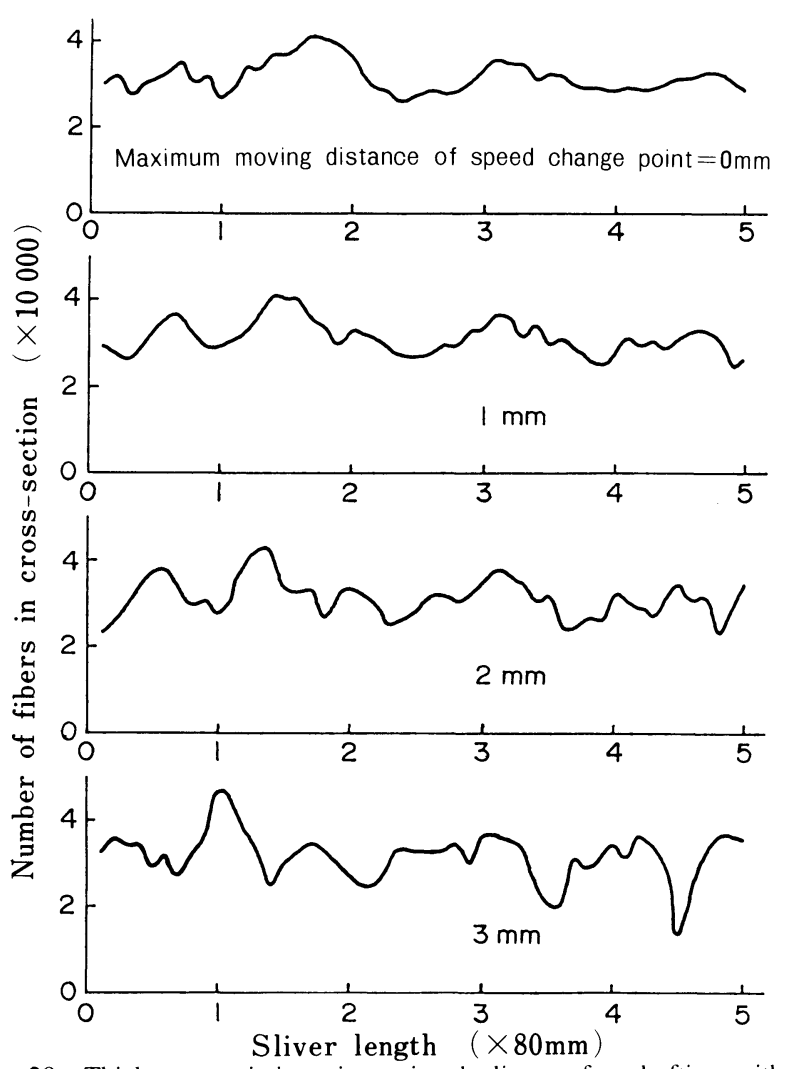

Fig. 20 Thickness variations in optional slivers after drafting with length increments not subdivided (Number of doublings $=8$, Draft distribution $=2.5 \times 8 / 2.5$ )
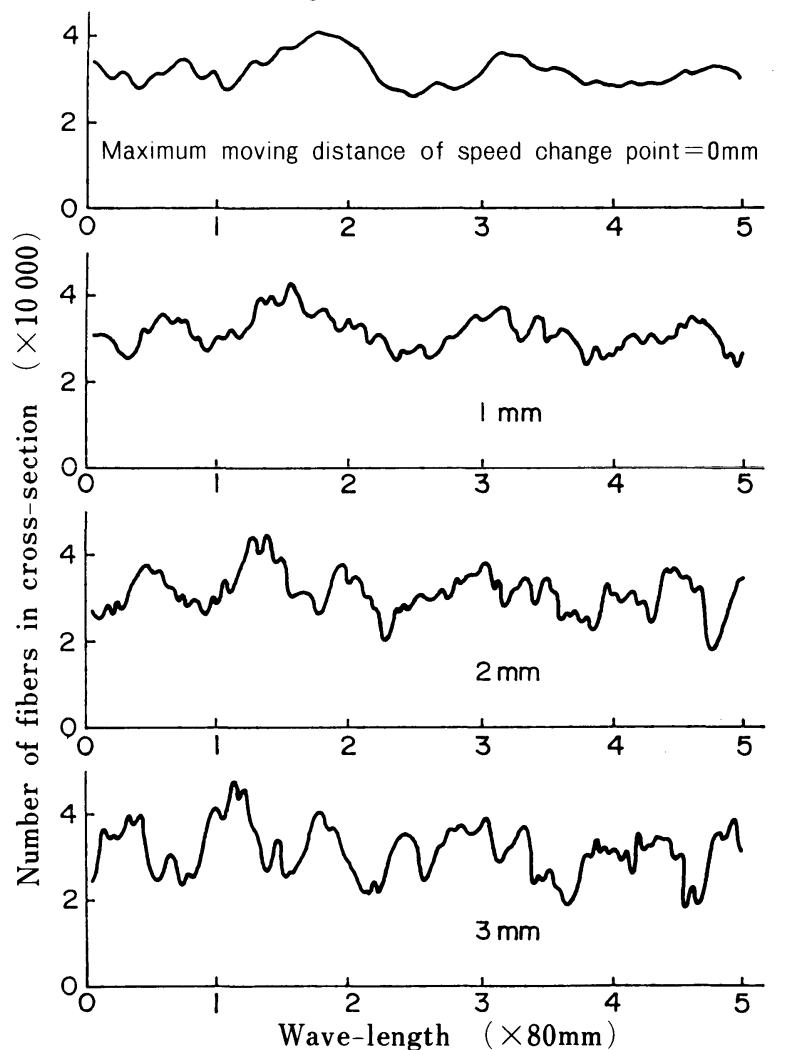

Fig. 21 Thickness variation in random slivers after drafting with length increments subdivided after the first drafting

(Number of doublings $=8$, Draft distribution $=2.5 \times 8 / 2.5$ )

Journal of The Textile Machinery Society of Japan 


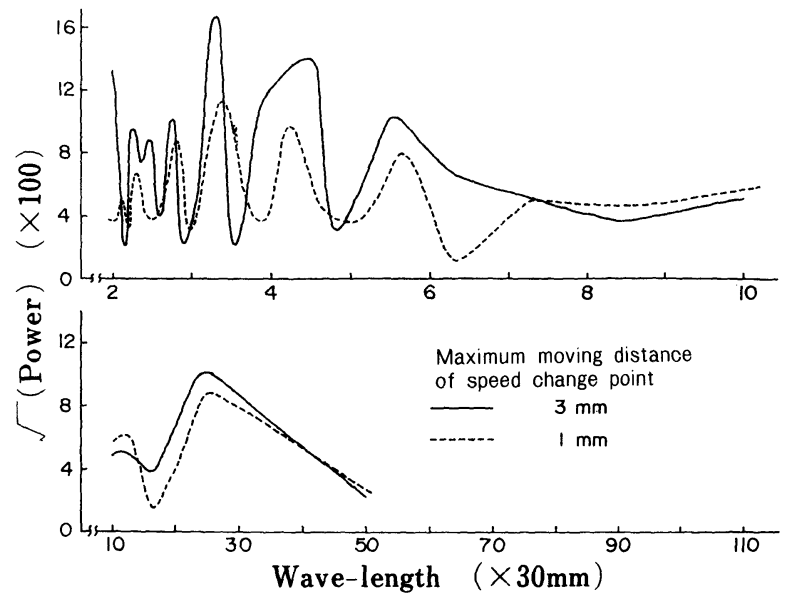

Fig. 22 Square root power spectrum of optional sliver after drafting (Length increments not subdivided) (Number of doublings $=8$, Draft distribution $=2.5 \times 8 / 2.5$ )
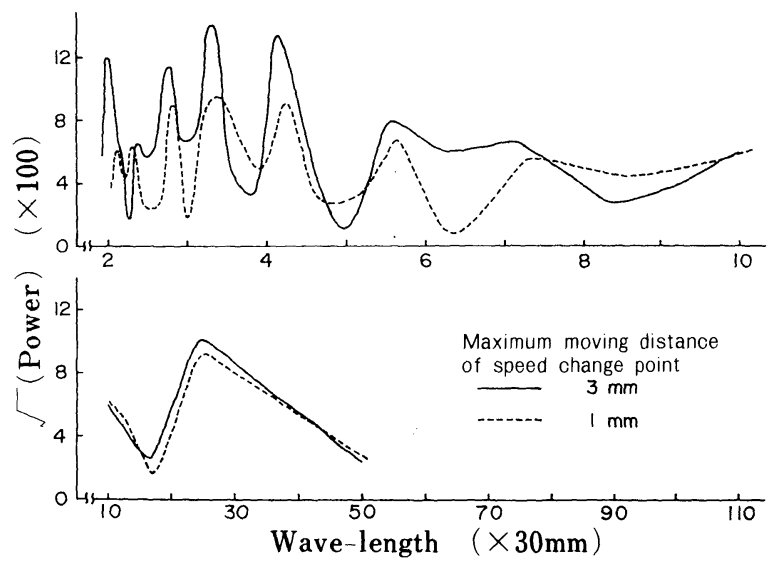

Fig. 23 Square root power spectrum of optional sliver after drafting (Length increments subdivided) (Number of doublings $=8$, Draft distribution $=2.5 \times 8 / 2.5$ )

the draft distribution at which the minimum variation occurs.

These results show that subdivision of $Z W$ has little effect when the total draft ratio less than about 8 .

\section{Conclusions}

If optional slivers or random slivers undergo a certain number $\boldsymbol{P}$ of doubling action, the coefficient of variation of doubled sliver is $1 / \sqrt{P}$ times the undoubled value to comply

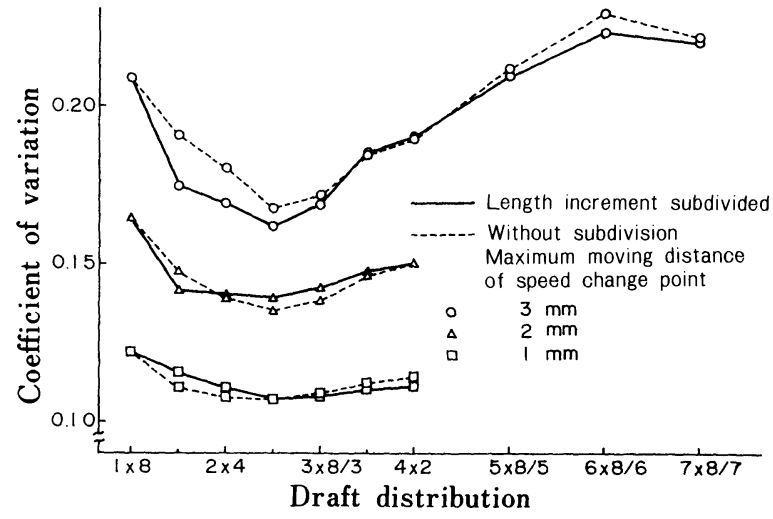

Fig. 24 Coefficient of variation of optional sliver versus draft distribution computed with and without subdivision of length increments

(Number of doublings $=8$, Total draft ratio $=8$ )

with the theory. But when the doubled sliver is drafted by a total ratio $P$, the coefficient of variation becomes more than that of the original undoubled sliver except when the drafting is ideal.

However, it is possible to minimize this value by selecting a proper draft distribution among the two draft zones. Futhermore, the minimized coefficient of variation increases with increasing number of doublings and total draft ratio.

Fourier analysis revealed that doubling and drafting increases unevenness in the short wave-length range of about two or three times the staple length, and decreases unevenness in the long wave-length range in optional slivers. This is not altogether in contradiction to the generally accepted fact that increased number of doublings make the drafted sliver more even.

\section{Literature Cited}

[1] J. R. Corley; Text. Ind., 110 (1949-3).

[2] J. R. Corley, J. Simpson; Text. Ind., 125 (1952-12).

[3] J. R. Corley, J. Simpson; Text. Ind., 114 (1956-12).

[4] Uchida, Takizawa, Tabata, Kobayashi and Noguchi; $J$. Japan Cot. Tech. Inst., 24 (1948-10).

[5] K. Fujino et al.; "theory and practice of the variation of thickness", Text. Mach. Soc. Japan, (1965).

[6] K. Fujino et al.; "theory and practice of drafting", Text. Mach. Soc. Japan, (1958). 